\title{
Hexagonal dielectric resonators and microcrystal lasers
}

\author{
Jan Wiersig \\ Max-Planck-Institut für Physik komplexer Systeme, D-01187 Dresden, Germany
}

(Dated: November 4, 2018)

\begin{abstract}
We study long-lived resonances (lowest-loss modes) in hexagonally shaped dielectric resonators in order to gain insight into the physics of a class of microcrystal lasers. Numerical results on resonance positions and lifetimes, near-field intensity patterns, far-field emission patterns, and effects of rounding of corners are presented. Most features are explained by a semiclassical approximation based on pseudointegrable ray dynamics and boundary waves. The semiclassical model is also relevant for other microlasers of polygonal geometry.

PACS numbers: 42.55.Sa, 05.45.Mt, 42.60.Da
\end{abstract}

\section{INTRODUCTION}

A novel class of microlasers based on nanoporous molecular sieve host-guest systems has been fabricated recently by Vietze et al. [1]. Organic dye guest molecules were put into the channel pores of a zeolitic microcrystal $\mathrm{AlPO}_{4}-5$ host. The aluminophosphate-crystals grow with natural hexagonal boundaries with a high degree of perfection. In terms of pump energy needed to reach lasing threshold these microlasers can compete with semiconductor based vertical-cavity surface-emitting lasers. This makes them a promising candidate for future applications as e.g. optical communication devices.

Microlasers and microresonators are not only relevant for experiments and applications but they are also of great interest from a theoretical point of view because they are open (or leaky) and they can be mesoscopic. In our case the latter depends on the lasing dye and on the crystal size. Both introduce a characteristic length scale: the wavelength $\lambda$ ranging from $600 \mathrm{~nm}$ to $800 \mathrm{~nm}$, and the side length $R$ of the hexagonal cross section of the crystal ranging from $2.6 \mu \mathrm{m}$ to $4.6 \mu \mathrm{m}$ in Refs. [1, 2]. In current experiments, larger crystals with $R$ up to $20 \mu \mathrm{m}$ are under investigation. Only the ratio of these two length scales is relevant. It can be expressed by the dimensionless size parameter $20 \leq k R \leq 190$, where $k=2 \pi / \lambda$ is the freespace wave number. For small $k R$ the wavelength and the cavity size are of the same order. The system is microscopic. For large but finite $k R$ the system is mesoscopic.

The theoretical analysis of the lasing modes reduces to the two-dimensional problem of resonant modes in a passive dielectric microresonator of regular hexagonal geometry (ignoring surface roughness). In Ref. [2] preliminary numerical computations of the full wave equations have been restricted to the near-field intensity pattern. We present a systematic numerical analysis of the hexagonally shaped dielectric resonator with $20 \leq k R \leq 60$, including resonance positions and lifetimes, near-field intensity patterns, far-field emission patterns, and effects of rounding of corners. To avoid convergence problems

*Electronic address: jwiersig@mpipks-dresden.mpg.de at corners, which had been a problem in Ref. [2], we will employ the boundary element method (BEM) [3].

To study the deep mesoscopic regime $k R>60$ we will introduce a semiclassical ray model. The semiclassical (short-wavelength) approximation is applied in the field of quantum chaos to relate quantum (wave) dynamics to their underlying classical (ray) dynamics. Most research efforts have been focused on closed resonators, socalled billiards, where the dielectric interface is replaced by a hard wall on which the wave function vanishes. The classical dynamics is free motion inside the billiard with specular reflections at the boundary.

The regular hexagon belongs to the class of rational polygonal billiards. All angles $\phi_{j}$ between sides are rationally related to $\pi$, i.e. $\phi_{j}=m_{j} \pi / n_{j}$, where $m_{j}, n_{j}>0$ are relatively prime integers. If all $m_{j}$ are equal to unity, for example in the case of the rectangle, then the dynamics is integrable. In the presence of critical corners with $m_{j}>1$ the dynamics is not integrable but instead pseudointegrable [4]. As for integrable systems the phase space is foliated by two-dimensional invariant surfaces [5, 6]. However, there are some peculiar features that distinguish these billiards strongly from integrable ones (and also from fully or partially chaotic billiards): (i) an invariant surface does not have the topology of a torus but instead that of a surface of higher genus [4], loosely speaking, a torus with additional handles; (ii) the dynamics is not quasiperiodic. This is reflected, for example, by multifractal Fourier spectra of classical observables [7, 8]; (iii) the quantum-classical correspondence is exotic $[9]$; (iv) the quantum spectrum obeys critical statistics 10. To compute the quantum spectrum of a pseudointegrable billiard with a semiclassical treatment is extremely difficult, if possible at all. In the present paper, we will demonstrate that for a sufficiently open hexagonal dielectric resonator the spectrum and the eigenmodes can be computed semiclassically. As a byproduct, we gain an intuitive understanding of the numerical results.

The paper is organized as follows. The system is defined in Sec. III. Section III presents the numerical analysis. The semiclassical ray model is introduced in Sec. IV. Finally, Sec. V contains our conclusions. 


\section{THE SYSTEM}

The numerical computation of lasing modes in a cavity with active medium and complex geometry is an extremely difficult task; see Refs. [11, 12]. Focusing on the effects of the geometry we here restrict our calculations to resonant modes in a passive cavity. Which of these resonant modes contribute to lasing is not important in our case since all long-lived resonant modes have very similar properties as we will see in the following.

In the experiments on the microcrystals it has been shown that the electromagnetic field is TM polarized [1, 2]. Maxwell's equations reduce to a two-dimensional wave equation 13

$$
-\nabla^{2} \psi=n^{2}(\mathbf{r}) k^{2} \psi
$$

with wave number $k$ and piece-wise constant index of refraction $n(\mathbf{r})$. The index of refraction is $n=1.466$ inside the cavity and 1 outside. The origin of the coordinate system $\mathbf{r}=(x, y)=(r \cos \theta, r \sin \theta)$ is located in the center of the hexagonal cavity. The complex-valued wave function $\psi$ represents the $z$-component of the real-valued electric field vector $E_{z}(\mathbf{r}, t)=\operatorname{Re}[\psi(\mathbf{r}) \exp (-i \omega t)]$ with $i^{2}=-1$, angular frequency $\omega=c k$ and speed of light in vacuum $c$. $\psi$ and $\nabla \psi$ are continuous at the boundary of the resonator. To model the situation in a laser, we impose the outgoing-wave condition

$$
\psi \sim h(\theta, k) \frac{\exp (i k r)}{\sqrt{r}}
$$

for large $r$. With a real-valued $n$ (passive resonator without absorption) this leads to modes that are exponentially decaying in time. The lifetime $\tau$ of these socalled resonant modes or short resonances is given by $\tau=-1 / 2 c \operatorname{Im}(k)$ with $\operatorname{Im}(k)<0$. The lifetime $\tau$ is related to the quality factor $Q=\operatorname{Re}(\omega) \tau$. The resonant modes are connected to the peak structure in scattering spectra; see e.g. Ref. [14]. We are here only interested in long-lived resonances that provide a sufficiently long lifetime for the light to accumulate the gain required to overcome the lasing threshold. Note that extremely longlived resonances are not relevant for lasers because they do not supply enough output power.

\section{A. Symmetry considerations}

Figure 1 shows the eight symmetry classes of the hexagon; see e.g. Ref. 15. In the notation $-+a, \ldots$, the first sign is + if the wave function is even with respect to $x \rightarrow-x$, and - otherwise. Correspondingly, the second sign refers to $y \rightarrow-y$. The letter $a$ indicates two symmetry lines, whereas the letter $b$ indicates six symmetry lines.

The $+-a$ and the $-+a$-modes have exactly the same complex $k$. The reason is that a $+-a$-mode can be converted to $\mathrm{a}-+a$-mode and vice versa in the following

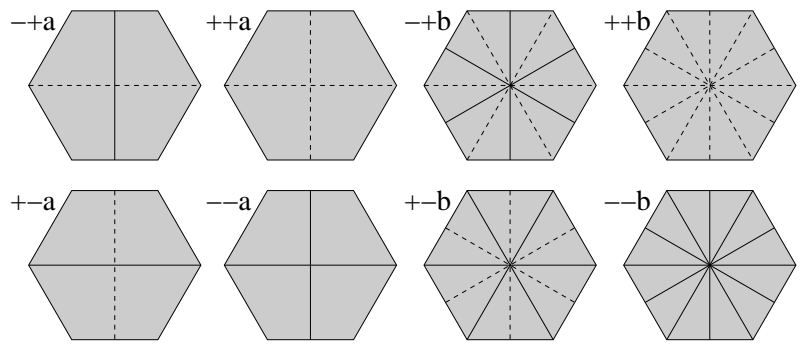

FIG. 1: The eight symmetry classes of the hexagon. Odd (Even) symmetry is marked by solid (dashed) lines.

way. Take two copies of a single $+-a$-mode. Rotate the first copy by $60^{\circ}$ clockwise around the origin of the coordinate system and the second copy by the same angle counterclockwise. Subtracting the two gives a $-+a$-mode with the same $k$. The way from $-+a$ to $+-a$, from $++a$ to $--a$, and from $--a$ to $++a$ is analogue. Hence, $a$-modes always appear in degenerate pairs having the same $k$.

Each linear superposition of such a pair of degenerate $a$-modes, we denote them by $\psi_{1}$ and $\psi_{2}$, is also a solution of the wave equation (11). Because of the 6-fold symmetry of the system, we can find always two superpositions $\psi_{ \pm}=\psi_{1}+p_{ \pm} \psi_{2}$ with the property that the corresponding intensities $\left|\psi_{+}\right|^{2}$ and $\left|\psi_{-}\right|^{2}$ are invariant under $60^{\circ}$ rotations. That means $\psi_{+}$and $\psi_{-}$change only by a phase factor $\exp i \Omega_{ \pm}$when rotated in real space by $60^{\circ}$. A full rotation about $360^{\circ}$ does not change the wave function. Hence, $\Omega_{ \pm}=\pi q / 3$ with $q=-2,-1,0,1,2,3$. Yet, $q=0$ and $q=3$ are not allowed because the intensities of $a$ modes are not invariant under $60^{\circ}$-rotations. A rotation about $180^{\circ}$ is identical to $(x, y) \rightarrow(-x,-y)$. From this we find $\Omega_{ \pm}= \pm \pi / 3$ for $\psi_{ \pm}$formed by $a$-modes of type ,+--+ and $\Omega_{ \pm}= \pm 2 \pi / 3$ for $\psi_{ \pm}$formed by $a$-modes of type,++-- . These requirements lead to

$$
p_{ \pm}=-\frac{\exp \left(i \Omega_{ \pm}\right) \psi_{1}(\mathbf{r})-\psi_{1}(\tilde{\mathbf{r}})}{\exp \left(i \Omega_{ \pm}\right) \psi_{2}(\mathbf{r})-\psi_{2}(\tilde{\mathbf{r}})}
$$

where $\mathbf{r} \neq(0,0)$ is an arbitrary point and $\tilde{\mathbf{r}}$ is $\mathbf{r}$ rotated by $60^{\circ}$ counterclockwise. Formula (3) is valid for any relative phase of $\psi_{1}$ and $\psi_{2}$.

The $b$-modes cannot be converted into each other since each $b$-mode is invariant under $60^{\circ}$-rotations up to a phase. Hence, $b$-modes do not form degenerate pairs.

\section{B. Rounding}

For our numerics it will be necessary to round the corners of the resonator slightly as depicted in Fig. 2. In terms of polar coordinates the parametrization of the boundary reads

$r^{s}=\frac{2 R^{s}}{\left(\cos \theta-\frac{1}{\sqrt{3}} \sin \theta\right)^{s}+\left(\frac{2}{\sqrt{3}} \sin \theta\right)^{s}+\left(\cos \theta+\frac{1}{\sqrt{3}} \sin \theta\right)^{s}}$ 
where $s$ is the rounding parameter, a positive even integer. $s=2$ gives the circle, whereas $s \rightarrow \infty$ gives the hexagon with flat sides and sharp corners. The parametrization in Eq. (1) preserves the full symmetry of the problem. Note that the parametrization used in Ref. [2] is different.

a)

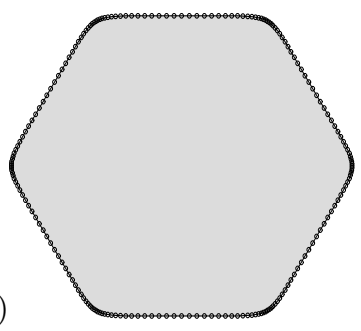

b)

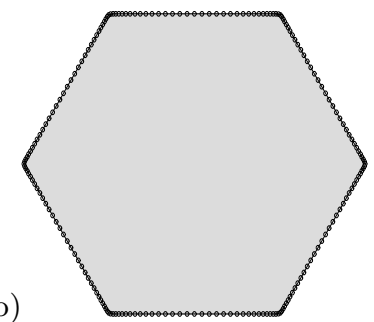

FIG. 2: Shape and discretization (circles) of the resonator with (a) $\mathrm{s}=20$, (b) $\mathrm{s}=200$ and $\eta=0.1$. For illustrational reasons only $N=200$ discretization points are used.

It is easy to show that the maximum curvature for the rounded hexagon in Eq. (伍) is given by $(s-1) / 3 R$ for $s \geq 6$. The radius of curvature $\rho$ per wavelength $\lambda=2 \pi / \operatorname{Re}(k)$ is therefore given by

$$
\frac{\rho}{\lambda}=\frac{3}{2 \pi} \frac{\operatorname{Re}(k R)}{s}
$$

for large $s$.

\section{NUMERICAL ANALYSIS}

A frequently used numerical method to solve wave equation (11) with outgoing-wave condition (2) is the wave- or mode-matching method 16]. The wave function is expanded in integer Bessel functions inside the cavity and in Hankel functions of first kind outside, so that the outgoing-wave condition (2) is fulfilled automatically. The Rayleigh hypothesis asserts that such an expansion is always possible. However, it can fail for geometries which are not sufficiently weak deformations of a circular cavity [17. So, using the mode-matching method for the hexagonal resonator in Ref. [2] is questionable. And indeed, according to Ref. [18], serious convergence problems appear in this situation. To avoid this problem we apply the BEM [3]. Exploiting Green's identity, the two-dimensional differential equation (II) is replaced by a one-dimensional integral equation defined only along the boundary of the cavity. To be slightly more precise, the boundary appears twice in the formalism: one bounds the interior of the cavity and one bounds the exterior. The boundaries are then discretized by dividing them into $2 N$ small boundary elements. For the boundary (4) we choose the following discretization

$$
\theta=\xi+\eta \sin [6(\xi+\pi / 2)] .
$$

The discretization points on the circle $\xi \in[0,2 \pi)$ are chosen to be spaced equidistantly. The parameter $\eta$ determines the distribution of points on the cavity (the shape of which is completely determined by the rounding parameter $s) . \quad \eta=0$ gives an uniform density of points while $\eta>0$ gives an enhancement near the corners as illustrated in Fig. 2. We always use $\eta=0.1$.

\section{A. Resonance positions in the complex plane}

Figure 3 shows the total cross section $\sigma$ as function of the dimensionless wave number $k R[\operatorname{Im}(k R)=0]$ for plane-wave scattering with incidence angle $\theta=15^{\circ}$. For numerical details of how to compute this quantity see Ref. [3]. We observe a single-mode spectrum, a series of roughly equidistant peaks (the fine structure around $k R \approx 24.5$ is an artifact of the BEM which can be removed by increasing the number of discretisation points [3]). We will count the peaks by the mode index (or quantum number) $m$ according to increasing $k R$ starting with $m=0$ at $k R=0$. The first peak at $k R \approx 20.5$ in Fig. 3 is then labeled by $m=23$. The position of a given peak $k R$ and its width $\Gamma$ in Fig. 3 are related to the complex value of $k R_{\text {mode }}$ of the corresponding resonant mode by means of $k R_{\text {mode }} \approx k R-i \Gamma / 2$. The subscript "mode" will be dropped from now on.

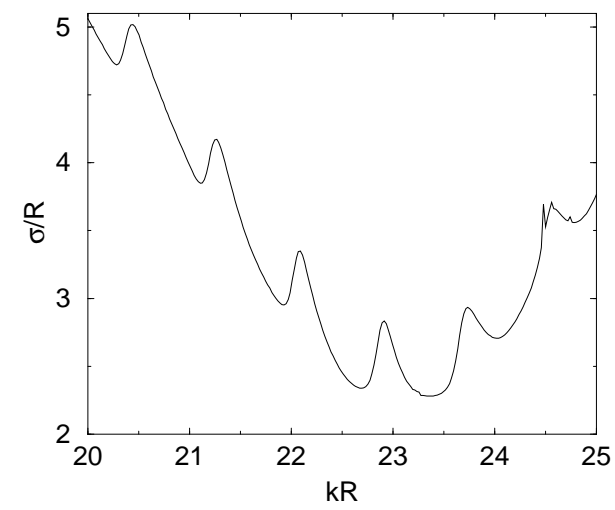

FIG. 3: Calculated total scattering cross section $\sigma / R$ vs. $k R$ for a hexagonal resonator. The plane wave is incidence at $15^{\circ}$ to the horizontal side faces. $s=100$, and $2 N=2000$.

The BEM not only solves the scattering problem but it also can compute the resonant modes. In Fig. 4 we plot the real and imaginary part of $k R$ of a resonance as function of the rounding parameter $s$. $\operatorname{Re}(k R)$ saturates around $s=55$, whereas $\operatorname{Im}(k R)$ saturates at $s \geq 100$. We take $s=100$ translating to $\rho / \lambda \approx 0.11$. It is surprising that one has to decrease $\rho$ to values one order of magnitude smaller than the wavelength. In the following we will fix $\rho / \lambda \approx 0.11$, that means when we change $\operatorname{Re}(k R)$, we have to change $s$ accordingly.

Figure 5 shows the long-lived resonances in the complex plane inside the strip $20 \leq \operatorname{Re}(k R) \leq 60$. Our numerics cannot cover the full regime of the microlaser experiments [1, 2] $20 \leq \operatorname{Re}(k R) \leq 190$. Small values of $|\operatorname{Im}(k R)|$ correspond to long-lived modes and, correspondingly, large values correspond to short-lived modes. 


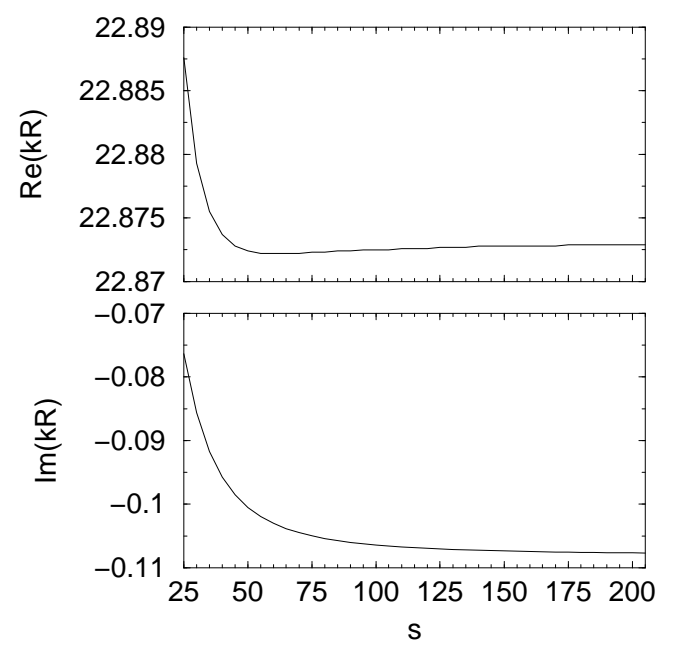

FIG. 4: Real and imaginary part of $k R$ as function of the rounding parameter $s .2 N=2000, m=26, k R=22.8725-i 0.1064$ for $s=100$.

Four features in Fig. 5 are striking: (i) the $a$-modes are two-fold degenerated as predicted from the symmetry considerations. (ii) The $b$-modes come in quasidegenerated pairs with slightly different $k R$ (differences in $\operatorname{Re}(k R)$ and $\operatorname{Im}(k R)$ are of the same order which cannot be seen in Fig. 5). It is not possible to resolve these small splittings in the scattering cross section in Fig. 3. (iii) The values of $\operatorname{Re}(k R)$ are approximately equidistantly spaced. (iv) Highly-excited resonances with $\operatorname{Re}(k R) \geq 34$ lie approximately on a smooth curve.

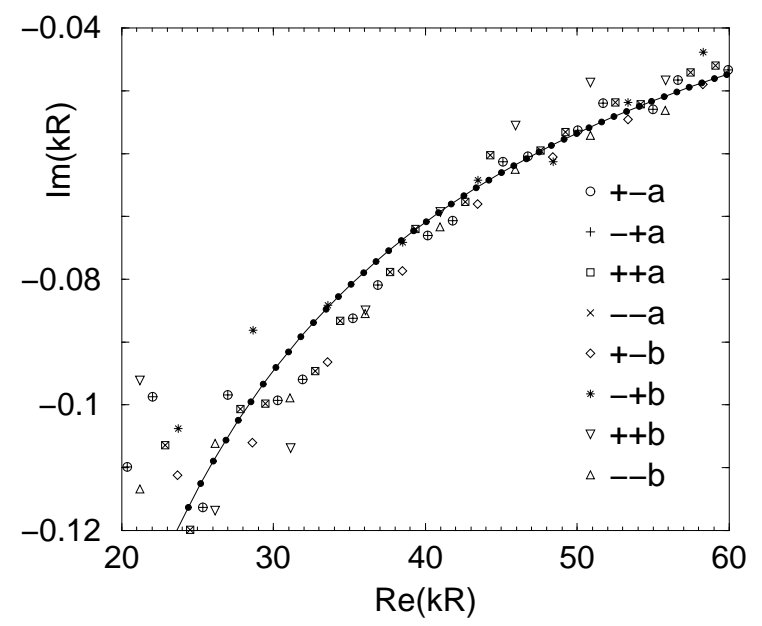

FIG. 5: Long-lived resonances in the complex plane, cf. Fig. 1. Filled circles on the solid line are the semiclassical solutions in Eqs. (17) and 24) from Sec. IV.

The equidistant spacing of $\operatorname{Re}(k R)$ can be seen more clearly in Fig. 6(a). Pairs of (quasi-) degenerated modes are labeled by the mode index. We see that all pairs lie extremely close to a line $\operatorname{Re}(k R)=\nu\left(m+m_{0}\right)$ with mode spacing $\nu$ and shift $m_{0}$. Linear regression gives $\nu \approx$ 0.8238 and $m_{0} \approx 1.7516$. The same mode spacing can be observed in Fig. 3. The corresponding free spectral range
$\Delta \lambda=\nu \lambda^{2} / 2 \pi R$ is in agreement with the experiments [1, 2].
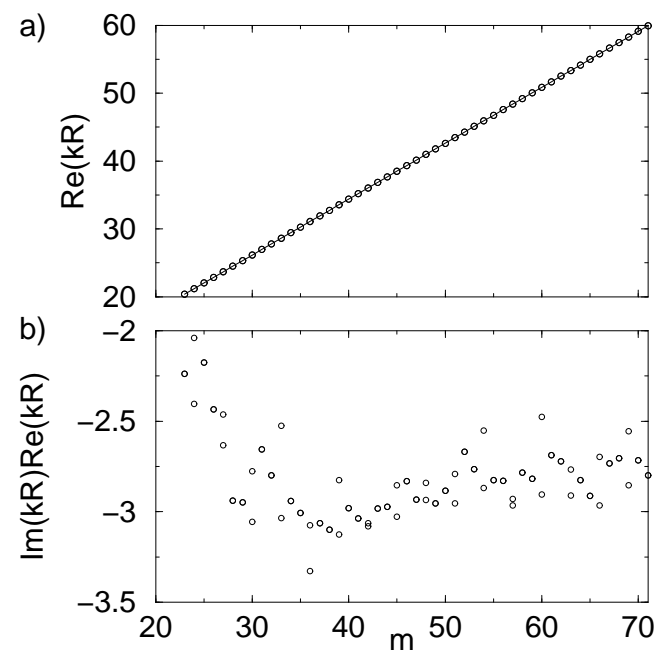

FIG. 6: (a) Wave number $\operatorname{Re}(k R)$ vs. mode index $m$. The data can be approximated by a straight line $\operatorname{Re}(k R)=\nu\left(m+m_{0}\right)$ with $\nu \approx 0.8238$ and $m_{0} \approx 1.7516$. (b) $\operatorname{Im}(k R) \operatorname{Re}(k R)$ vs. $m$. For $m \geq 34$, the data is well approximated by the mean value $\approx-2.87$.

From Fig. 6(b) we can infer that the product of real and imaginary part of $k R$ for highly-excited modes does not depend on the mode index $m$. We find

$$
\operatorname{Im}(k R) \operatorname{Re}(k R) \approx-2.87
$$

for $n=1.466$. Later we will see that this remarkable relation is a good approximation not only in the numerical accessible regime but also in the full experimental regime. Relation (7) implies that the lifetime is proportional to $\operatorname{Re}(k) R^{2}$ and the quality $Q$ is proportional to $\operatorname{Re}(k R)^{2}$ ( $Q$ ranges from roughly 70 to 6400 ). This finding is relevant for the future experiments on microcrystal lasers: it implies that the laser threshold decreases as the size of the resonator is increased.

Due to the qualitative change in behaviour around $\operatorname{Re}(k R) \approx 34$ in Figs. . and 6(b) we distinguish the regions $\operatorname{Re}(k R)<34$ and $\operatorname{Re}(k R)>34$. We refer to the former one as the microscopic regime and to the latter as the mesoscopic regime.

\section{B. Mode structure}

Figures $7(\mathrm{a})$ and (b) show the near-field intensity pattern of the resonances $26++a$ and $26--a$ (mode index $m=26$ ), respectively. While these two resonances are standing waves (ignoring the uniform temporal decay), the corresponding superpositions $\psi_{+}$and $\psi_{-}$in Figs. If(c) and (d) are unidirectional traveling waves. We call them chiral modes. Such a mode is specified by its mode index $m$ and a label + (traveling counterclockwise) or (traveling clockwise). In the following we will deal only 
with these chiral modes which are more straightforward to compare to the ray dynamics. We remark that the Husimi function representation frequently applied to optical microcavities (see e.g. Ref. [19]) does not provide more insight in our case. a)

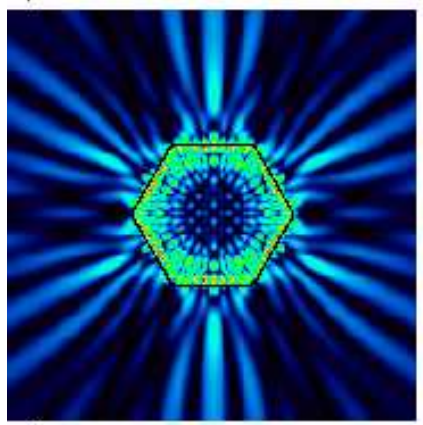

c)

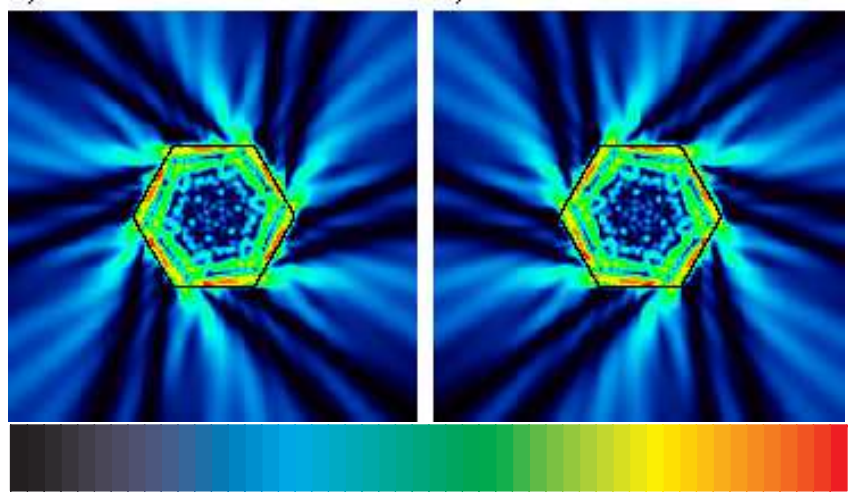

b)

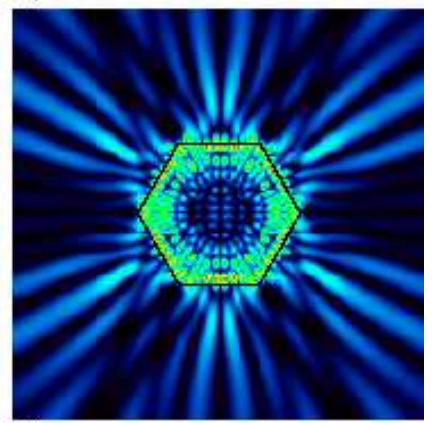

d)

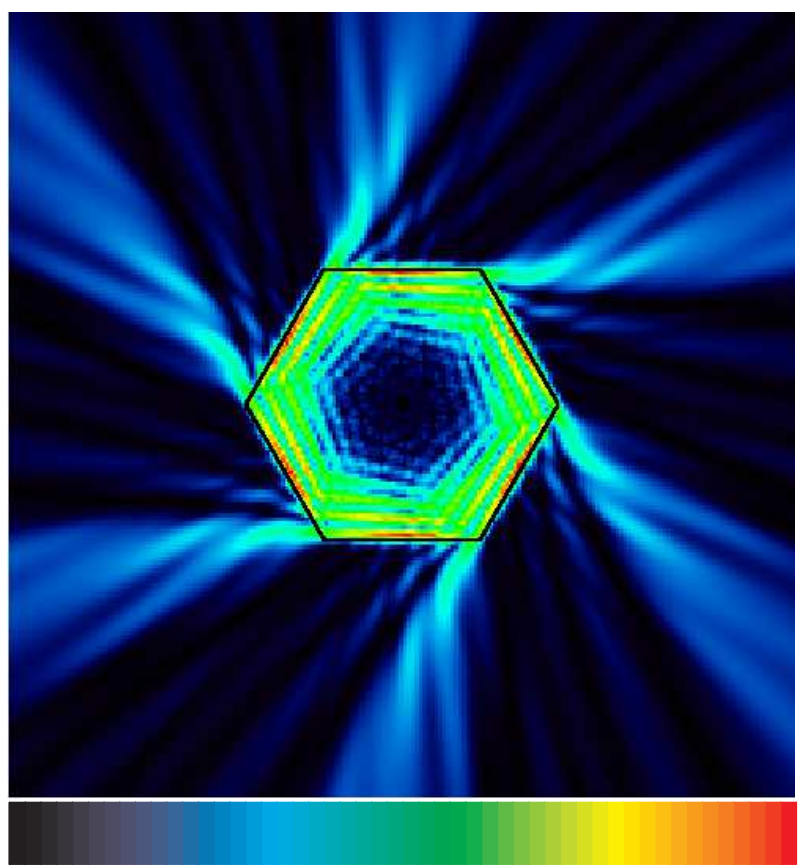

FIG. 8: Chiral resonance 50-. $k R=42.6318-i 0.06766, s=200$, $2 N=4000$.

intensity directions are now parallel to the edges. The latter fact can be seen better in the far-field emission pattern in Fig. 11.

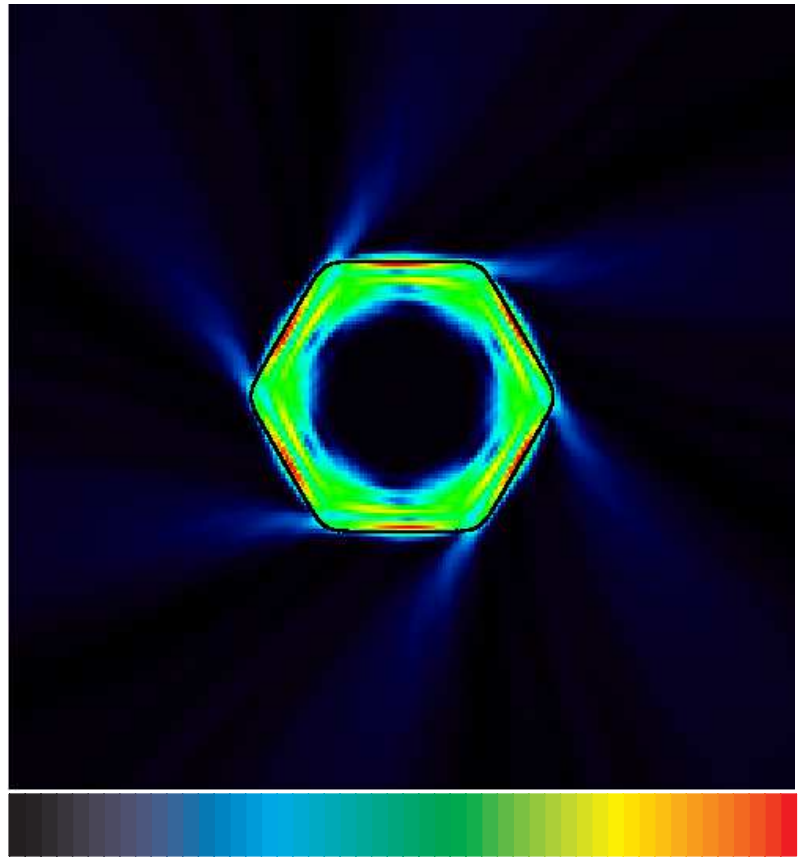

FIG $_{0}$ 9: Resonance 50- in a rounded hexagon with $s=20$, cf. Fig. 8. $k R=42.7099-i 0.01836,2 N=4000$.
FIG. 7: Calculated near-field intensity pattern $|\psi(\mathbf{r})|^{2}$ of two-fold degenerated resonances. (a) $26++a$ and (b) $26--a$ with welldefined parity. (c) $26+$ and (d) 26- with well-defined chirality. Intensity is higher for redder colours, and vanishes in the dark regions. $k R=22.8725-i 0.1064, s=100,2 N=2000$.

Figure 8 shows a higher-excited superposition $\psi_{-}$. The following properties can be observed: (i) the intensity is concentrated along the boundary of the cavity, resembling whispering-gallery modes in circular or weakly deformed circular cavities. (ii) The wave pattern looks regular. An approximate nodal-line structure with a peculiar twist is visible. (iii) The emission is predominantly at the corners. This is in agreement with the laser emission measured in the experiments in Refs. [1], 2]. (iv) Outside the cavity the light propagates along certain directions not parallel to the facets. The latter fact can be seen more clearly in the far-field distribution shown in Fig. 10. There are six emission peaks with angular width of $\approx 14^{\circ}$ and angular distance to the nearest facet of $\approx 17^{\circ}$.

The sensitivity to rounding found for the resonance positions in the complex plane, see Fig. 4, also shows up in the mode structure. Figure 9 shows the near-field intensity pattern of a rounded hexagon with $s=20$, i.e. $\rho \approx \lambda$. The emission is again at the corners, but it is reduced. Moreover, the directionality has decreased and the high- 

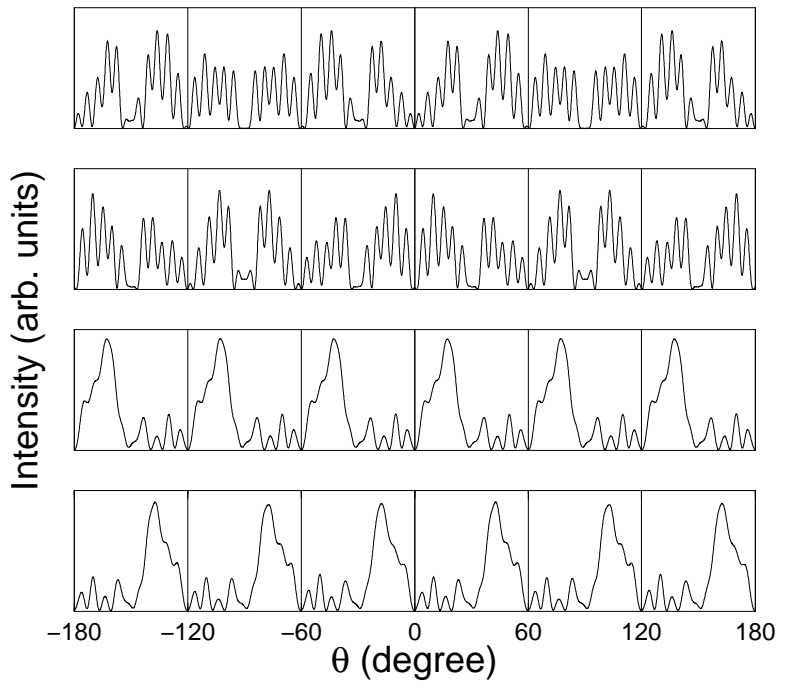

FIG. 10: Far-field emission pattern, $|\psi(r, \theta)|^{2}$ for large $r$, of (from above) $50--a, 50++a, 50-$, and $50+$ modes; cf. Fig. 8. Vertical lines mark the directions parallel to the edges.
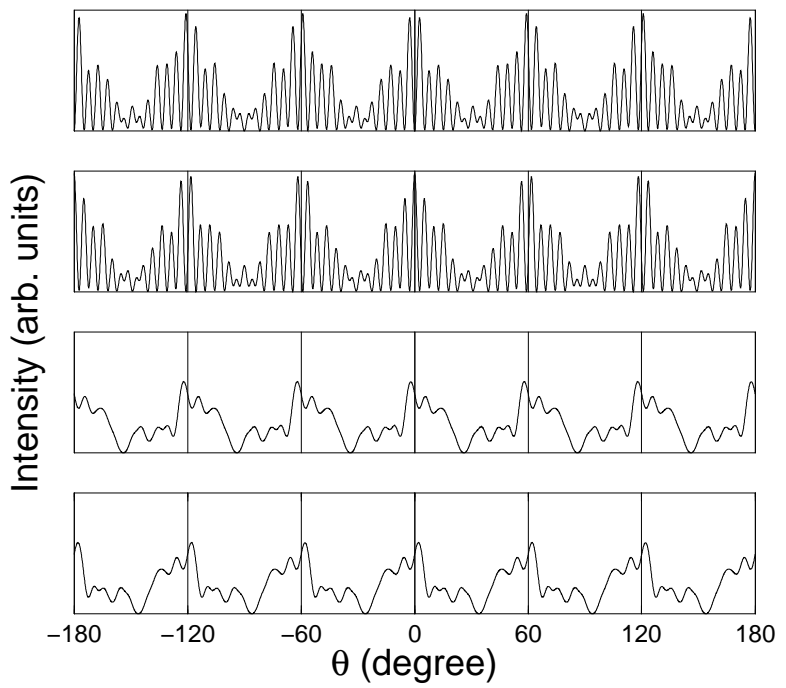

FIG. 11: Far-field emission pattern of the resonance (from above) $50--a, 50++a, 50-$, and $50+$ in the rounded hexagon; cf. Fig. 9 .

\section{SEMICLASSICAL ANALYSIS}

Having computed numerically the long-lived resonances in the regime $20 \leq \operatorname{Re}(k R) \leq 60$, we now introduce a semiclassical ray model in order to see what happens in the deep mesoscopic regime $\operatorname{Re}(k R)>60$. So far, semiclassical approximations of dielectric resonators have been discussed only for the case of smooth boundaries 16, 20, 21. Our heuristic approach is divided into three steps: geometric optics, semiclassical quantization and emission mechanisms.

\section{A. Geometric optics}

Geometric optics follows from wave equation (11) in the limit $\operatorname{Re}(k R) \rightarrow \infty$ neglecting all interference effects. In the following we focus on low-index materials with $n_{\min }<n<n_{\max }$ where $n_{\min }=1 / \sin 60^{\circ} \approx 1.16$ and $n_{\max }=1 / \sin 30^{\circ}=2$. The lower bound guarantees that a six-bounce periodic ray with angle of incidence $\theta_{i}=60^{\circ}>\theta_{c}\left(\right.$ with $\left.\sin \theta_{c}=1 / n\right)$ is trapped within the hexagonal resonator by total internal reflection at the facets; see Figs. 12 (some aspects of the much simpler case $n<n_{\text {min }}$ have been studied in Ref. [22]). The periodic ray is marginally stable with respect to shifting it along the boundary. In this way we obtain a whole family of periodic rays with identical length and angle of incidence.

The upper bound $n_{\max }=2$ ensures that triangularshaped periodic rays with $30^{\circ}$ angle of incidence are not totally reflected. The periodic-ray family with $\theta_{i}=60^{\circ}$ is then the only long-lived family of short period. Yet, since periodic rays are dense in phase space, we cannot exclude the possibility that there are also long-lived periodic rays of high period.

It should be emphasized at this point that the (infinite) long lifetime of the rays is relevant for our purpose, whereas the periodicity of the rays is not relevant. The latter comes in here simply because periodic rays (and their neighborhood) can be long-lived. We will not apply methods from semiclassical periodic-orbit theory [23].

The periodic rays are unstable with respect of changing the angle of incidence. Figure 12 shows a ray with slightly different initial angle of incidence. The ray is slowly diverging from the central one. After some time, it reaches the corner on its other side at (almost) normal incidence. Consequently, it then escapes refractively (with probability close to 1 ).

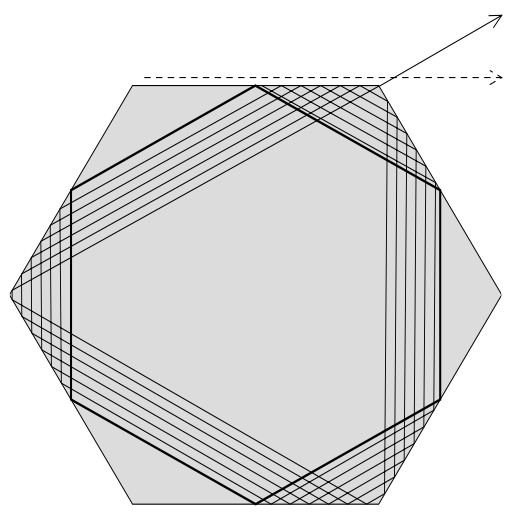

FIG. 12: Semiclassical ray model. Thick line marks a member of the family of long-lived rays, other members are obtained by shifting the ray along the boundary (not shown). The thin line marks a ray with slightly different angle of incidence. Arrows indicate emission due to pseudointegrable dynamics (thin) and boundary waves (dashed).

As long as the ray does not escape from the cavity 
it behaves as if it were in a closed resonator, i.e. in a billiard. It is illuminating to examine the ray dynamics of the open system in terms of the invariant surface of the hexagonal billiard. The genus of the invariant surfaces is given by the general formula [4]

$$
g=1+\frac{\mathcal{N}}{2} \sum_{j} \frac{m_{j}-1}{n_{j}}
$$

where the sum is over all corners with angles $\phi_{j}=$ $m_{j} \pi / n_{j}$ and $\mathcal{N}$ is the least common multiple of the $n_{i}$. For the hexagon $m_{j}=2$ and $n_{j}=3$ for all $j$; hence, $\mathcal{N}=3$ and finally $g=4$. The surface of genus four is shown schematically in Fig. 13. The topology is the same for all initial conditions. First, we consider the special case where the surface is foliated by periodic orbits. Each periodic ray with $\theta_{i}=60^{\circ}$ appears here as two disjoint circles with different sense of rotation. These two circles correspond to the two unidirectional traveling waves. In the following we will focus on the circle with clockwise rotational sense. On the same invariant surface we find also periodic rays of "bouncing-ball" type with angle of incidence $\theta_{i}=0^{\circ}$. In the open system, the bouncing-ball rays are not long-lived since they are not totally reflected.

The dynamics on a generic invariant surface in the neighborhood of the special surface discussed above is as follows. A nonperiodic ray starting near the central periodic ray with slightly different initial angle of incidence stays in the vicinity of the central ray by winding around the handle many times. Finally, it has to leave the vicinity of the periodic ray since pseudointegrable motion is ergodic on generic invariant surfaces [24]. Before the ray can reach another handle (in the grey region in Fig. 13) and perform complicated dynamics, it escapes refractively from the cavity. Hence, the sufficiently open resonator does not see the complicated topology of the full invariant surface but just two disjoint tori. In this sense, the openness moves the system closer to integrability. That is the reason why we can derive in the following subsection a practical semiclassical approximation for the open hexagonal resonator whereas this is impossible for the hexagonal billiard.

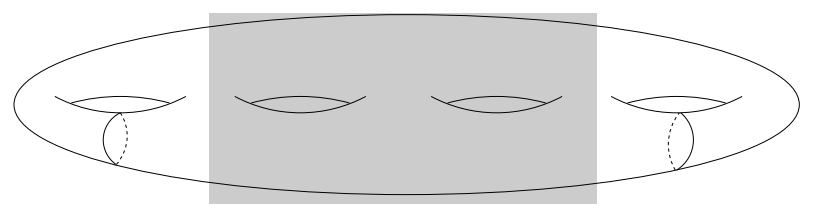

FIG. 13: Invariant surface of the hexagonal billiard. The small circle on the left (right) hand side represents the central periodic ray in Fig. 12 cycling (counter-) clockwise. In the grey region the condition for total internal reflection is not fulfilled.

\section{B. Semiclassical quantization}

Having identified the long-lived part of classical phase space, we now quantize it in a semiclassical approximation. This approximation is valid for small $1 / \operatorname{Re}(k R)$ and it does include interference effects, in contrast to the geometric-optics limit.

Because of the absence of stability of the periodic rays the conventional approach in laser optics, the paraxial approximation (see e.g. Ref. 25]), does not work. Nevertheless, the semiclassical quantization is simple. The idea is that an integer number $m=1,2,3, \ldots$ of wavelengths $\lambda_{\text {inside }}=\lambda / n=2 \pi / n \operatorname{Re}(k)$ fits on the path length of the long-lived ray shown in Fig. 12, taking into account the phase shifts at the dielectric boundary. It will turn out that the number $m$ defined in this way is identical to the mode index $m$ as used in Sec. III.

To compute the phase shifts we consider the text-book treatment of reflection of a plane wave at an infinitely extended dielectric interface; see e.g. Ref. [26]. This simplified setup is justified if $\lambda \ll R$, i.e. $\operatorname{Re}(k R) \gg 1$. We shift the origin of the coordinate system such that $y=0$ is the dielectric boundary bounding the lowerindex region from below and the higher-index region from above. In the higher-index region there is an incident wave

$$
\psi_{i}=A_{i} \exp \left[i \operatorname{Re}(k) n\left(x \sin \theta_{i}+y \cos \theta_{i}\right)\right],
$$

with amplitude $A_{i}$. We set $A_{i}=1$ without loss of generality. The reflected wave is given by

$$
\psi_{r}=A_{r} \exp \left[i \operatorname{Re}(k) n\left(x \sin \theta_{r}-y \cos \theta_{r}\right)\right] .
$$

In the lower-index region there is an emitted wave

$$
\psi_{e}=A_{e} \exp \left[i \operatorname{Re}(k)\left(x \sin \theta_{e}+y \cos \theta_{e}\right)\right] .
$$

The boundary conditions

$$
\psi_{i}(x, 0)+\psi_{r}(x, 0)=\psi_{e}(x, 0)
$$

and

$$
\left.\frac{\partial \psi_{i}}{\partial y}\right|_{(x, 0)}+\left.\frac{\partial \psi_{r}}{\partial y}\right|_{(x, 0)}=\left.\frac{\partial \psi_{e}}{\partial y}\right|_{(x, 0)}
$$

lead to $\theta_{i}=\theta_{r}$, Snell's law $n \sin \theta_{i}=\sin \theta_{e}, A_{i}+A_{r}=A_{e}$ and the Fresnel formula

$$
A_{r}=\frac{1-i \alpha}{1+i \alpha}
$$

with

$$
\alpha=\frac{\sqrt{n^{2} \sin ^{2} \theta_{i}-1}}{n \cos \theta_{i}} .
$$

The quantization (or resonance) condition then reads

$$
e^{i n \operatorname{Re}(k) l} A_{r}^{6}=1,
$$


with the length of the periodic rays $l=3 \sqrt{3} R$. After some algebraic manipulations we arrive at

$$
\operatorname{Re}(k R)=\frac{2 \pi}{3 \sqrt{3} n}(m+\beta)
$$

with the total boundary phase shift $\beta$ given by

$$
\tan \frac{\pi}{6} \beta=\alpha .
$$

The quantization condition (17) explains the singlemode spectrum in Figs. 3 and 6(a). With $n=1.466$ the mode spacing is $2 \pi / 3 \sqrt{3} n \approx 0.8248$ in agreement with $\nu=0.8238$ obtained by fitting to the numerical data. However, the shift $\beta \approx 1.562$ differs a bit from the numerically obtained shift $m_{0} \approx 1.7516$. To understand this discrepancy we plot in Fig. 14 the phase shifts $\beta_{m}$ defined as $\beta$ computed from Eq. (17) inserting the numerically computed values of $\operatorname{Re}(k \vec{R})$. Clearly, going towards the semiclassical limit $m \rightarrow \infty$ leaves the crude fitting value $m_{0} \approx 1.7516$ in favour of a smaller value $\beta_{\infty}=\lim _{m \rightarrow \infty} \beta_{m}$ closer to our semiclassical prediction $\beta \approx 1.562$.

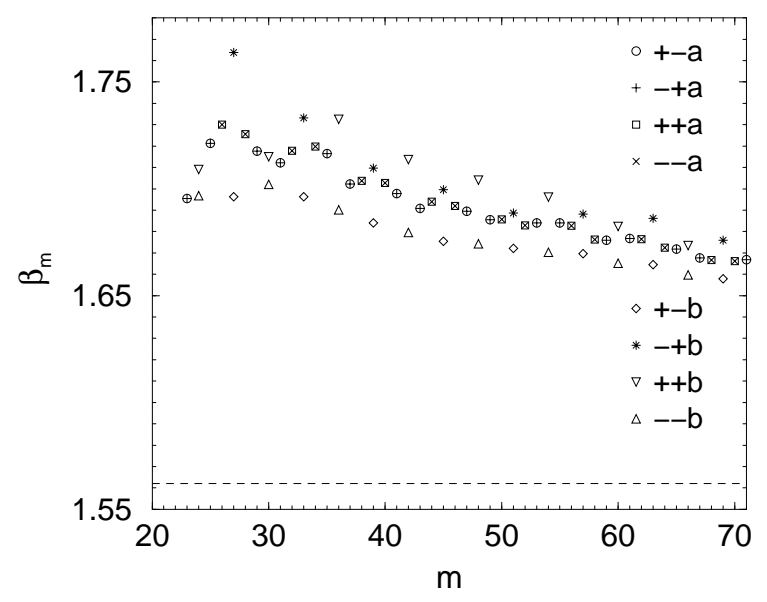

FIG. 14: $\beta_{m}$ vs. mode index $m$. Dashed line marks the semiclassical prediction $\beta \approx 1.562$.

Let us assume that $\beta_{m}$ has the following form

$$
\beta_{m}=\gamma m^{-\delta}+\beta_{\infty}
$$

and that $\beta_{\infty}$ equals our semiclassical solution $\beta \approx 1.562$. Figure 15 shows $\Delta \beta=\beta_{m}-\beta_{\infty}$ as function of $m \geq 34$ in a log-log plot for fixed $n=1.466$. Linear regression gives $\gamma \approx 0.89$ and $\delta \approx 0.5038$. Based on this numerical finding we conjecture that the next order in the semiclassical approximation (17) is of the form $\gamma / \sqrt{m}$.

Observation of Figs. If(c) and (d) shows that the "nodal lines" close to the boundary of the cavity are not exactly parallel to the boundary but slightly tilted (about $5^{\circ}$ ). This angular shift decreases as one goes to higherexcited modes. For example, it is not visible by eye in Fig. 8. Specular reflection of a plane wave at the boundary leads always to nodal lines parallel to the boundary.

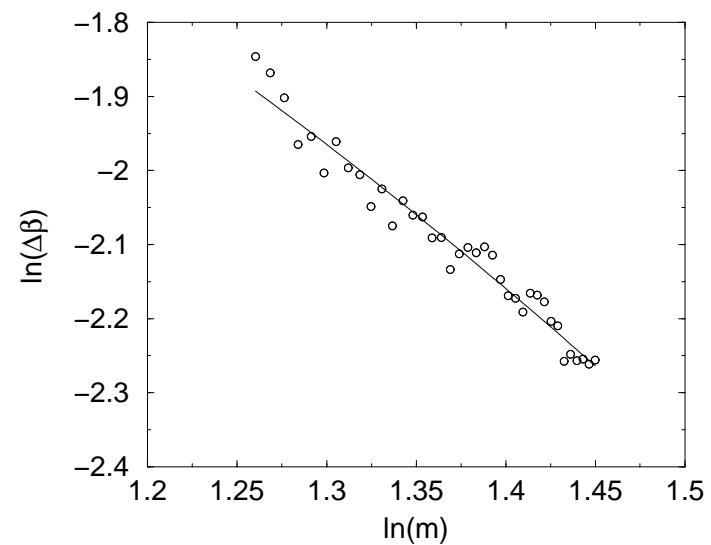

FIG. 15: $\ln \Delta \beta$ vs. $\ln m$ with $m \geq 34$. For quasi-degenerate modes the arithmetic mean of $\Delta \beta$ is taken.

Hence, it is reasonable to interpret the angular shift as a kind of non-specular reflection. The angular shift can be traced back to the fact that the wave function restricted to the boundary is a periodic function. Because of the periodicity, the Fourier spectrum of the wave function along the boundary is discrete. The discrete peaks can be related to a discrete set of allowed angles of incidence. The angle $\theta_{i}=60^{\circ}$ typically falls between two neighboring discrete angles. In Sec. A in the Appendix we compute the angular shift analytically to first order as $\Delta \theta=\theta_{i}-60^{\circ}=\theta_{i}-\pi / 3 \approx-\sqrt{3} \beta / m$ in agreement with the numerics (not shown). In the semiclassical limit $m \rightarrow \infty$ the angular shift vanishes. Our angular shift is different from those cases discussed in the context of non-specular reflections (see e.g. Ref. 27]), in that it happens above the critical angle of total internal reflection. Moreover, it has nothing to do with the angular Goos-Hänchen effect (see e.g. Ref. [28]), which is the Goos-Hänchen shift at a curved interface.

The number of wavelengths fitting on a path length determines the symmetry class. Straightforward considerations give Tab. If. The semiclassical quantization procedure neither distinguishes between +- and -+ parties nor between ++ and -- parties. Hence, within the semiclassical approximation these modes are exactly degenerated, regardless whether they are of type $a$ or $b$. This explains the numerical finding of quasi-degenerate $b$-modes.

The fact that our model predicts a single-mode spectrum, a one-parameter $m=1,2, \ldots$ family of long-lived resonances, is related to the fact that there is only one long-lived family of periodic rays (with short period). There is no "transversal mode index" which would be expected if the paraxial approximation were applicable. Higher transverse modes correspond to rays with angle of incidence considerably different from $60^{\circ}$. They are much shorter-lived which becomes clear in the following subsection when we discuss the emission mechanisms. 


$$
\begin{array}{|c|c|}
\hline m & \text { symmetry class } \\
\hline 1 & +-a,-+a \\
2 & ++a,--a \\
3 & +-b,-+b \\
4 & ++a,--a \\
5 & +-a,-+a \\
6 & ++b,--b \\
\hline
\end{array}
$$

TABLE I: Symmetry class and mode index $m$. The table is periodic in $m$ with period 6 .

\section{Emission mechanisms}

Having derived a semiclassical quantization condition for the real part of $k R$, we now compute its imaginary part. The temporal behavior of the intensity of a resonant mode is $I \propto \exp [2 \operatorname{Im}(\omega) t]$. The outgoing relative intensity per unit time is

$$
\Delta I=-\frac{1}{I} \frac{d I}{d t}=-2 \operatorname{Im}(\omega)=-2 c \operatorname{Im}(k) .
$$

What are the mechanisms for this decay of intensity? Obviously, there is no classical mechanism. In the framework of ray optics, the periodic rays in Fig. 12 remain forever in the cavity. Hence, we have to include explicitly wave effects. We identify three candidates of such wave effects, all of which are related to the corners. The first one is diffraction at corners. Corner diffraction may be a emission mechanism since a wave with finite wavelength coming close to a corner is diffracted partly to the exterior and partly back into the interior with another spectrum of directions for which the condition of total internal reflection may not be fulfilled at the next reflection. We will argue that corner diffraction is important for the emission directionality but not for the escape rate. For the escape rate we find two new relevant effects which are responsible for transport to corners. We call them boundary-wave leakage and pseudointegrable leakage.

The boundary-wave leakage is illustrated in Fig. 12. An evanescent boundary wave travels along an infinitely extended dielectric interface from $-\infty$ to $+\infty$. We assume that at a finite interface the boundary wave fully separates from the interface at the corner. In Sec. B in the Appendix we determine the outgoing relative intensity for any regular polygon (equilateral triangle, square, hexagon, ...) due to boundary waves. In particular, for the hexagon we find

$$
\Delta I_{\mathrm{bw}}=\frac{3 c}{4 \operatorname{Re}(k) R^{2}} \frac{n^{3}}{\sqrt{3 n^{2} / 4-1}\left(n^{2}-1\right)} .
$$

The pseudointegrable leakage is due to the fact that wave optics does not realize exactly $\theta_{i}=60^{\circ}$ as already mentioned and shown in Sec. A in the Appendix. Putting this small angular deviation $\Delta \theta$ into the initial conditions gives rise to rays with finite lifetime due to the pseudointegrable dynamics; see Fig. 12. In Sec. Q in the Appendix we estimate the outgoing relative intensity due to pseudointegrable leakage for the hexagon as

$$
\Delta I_{\mathrm{p}}=\frac{4 \pi c}{3 \operatorname{Re}(k) R^{2}} \frac{\beta(n)}{n^{2}} .
$$

The derivation can be easily extended to any regular polygon. Cavities with integrable internal dynamics, like the equilateral triangle and the square, have $\Delta I_{\mathrm{p}}=0$. That means the neighborhood of the long-lived family of periodic rays has roughly the same lifetime as the periodic rays. In such a case, we expect a multimode spectrum in the mesoscopic regime. Indeed, this has been found in scattering experiments on the dielectric square in Ref. 29].

Remarkably, both contributions in Eqs. (21) and (22) have the same dependence on $\operatorname{Re}(k)$. In either case, the contribution vanishes in the limit $\operatorname{Re}(k) \rightarrow \infty$, reflecting their wave nature. Adding both contributions,

$$
\Delta I=\Delta I_{\mathrm{bw}}+\Delta I_{\mathrm{p}},
$$

gives the central result

$$
\operatorname{Im}(k R) \operatorname{Re}(k R)=f(n)
$$

with

$$
\begin{gathered}
f(n)=f_{\mathrm{bw}}(n)+f_{\mathrm{p}}(n), \\
f_{\mathrm{bw}}(n)=-\frac{3 n^{3}}{8 \sqrt{3 n^{2} / 4-1}\left(n^{2}-1\right)},
\end{gathered}
$$

and

$$
f_{\mathrm{p}}(n)=-\frac{2 \pi}{3} \frac{\beta(n)}{n^{2}} .
$$

For $n=1.466$ we find $\operatorname{Im}(k R) \operatorname{Re}(k R) \approx-2.837$ in reasonable agreement with the numerical data in Figs. 5 and 6(b). Figure 16 compares the semiclassical result in Eqs. (24)-(27) to the numerical data as function of $n$. While some resonances are very well described by the semiclassical approximation, some are only roughly described. The latter cases correspond to the strong fluctuations around the semiclassical hyperbola (24) already visible in Fig. 司.

Our ray model also explains some aspects of the mode structure in Figs. 8 and 10. The whispering-gallery-like structure is related to the geometry of long-lived rays; see Fig. 12. The envelopes of the emission direction are described by the boundary-wave leakage and the pseudointegrable leakage; cf. Figs. 8, 10 and 12. The distribution within the envelopes is not predicted by our model. Here, corner diffraction is important because both the boundary waves and the pseudointegrable ray dynamics lead to escape arbitrarily close to the corner.

Our ray model gives a natural explanation for the sensitivity to rounding of corners. Let us assume that a sufficiently small rounding only slightly weakens the 


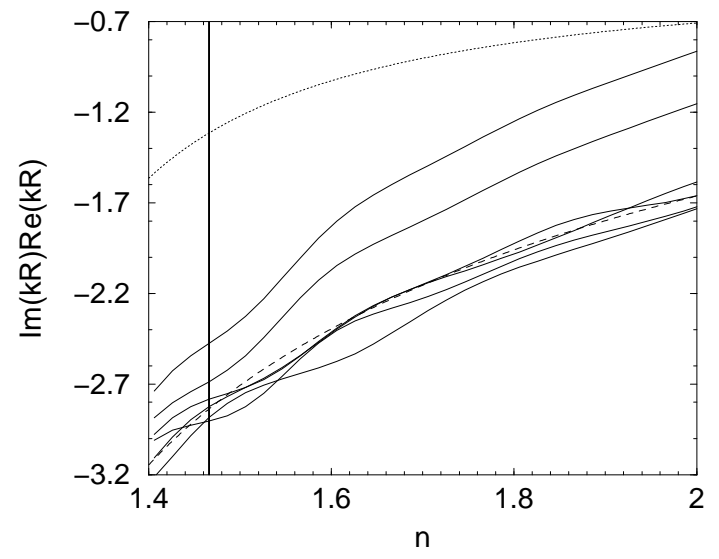

FIG. 16: $\operatorname{Im}(k R) \operatorname{Re}(k R)$ vs. $n$ for several resonances with $m \geq 50$ (solid curves). The contribution of the boundary waves, Eq. (26), is shown as dotted curve. The entire contribution, i.e. with pseudointegrable leakage, Eq. (27), is shown as dashed curve. The vertical line marks $n=1.466$.

loss due to boundary waves, whereas it reduces corner diffraction strongly. Numerical simulations of ray dynamics in rounded hexagons show that the periodic rays, see Fig. 12, are stabilized. This statement is independent on the particular chosen boundary parametrization. Hence, there is no pseudointegrable leakage. This leads to considerably larger lifetimes and emission parallel to the facets; compare Figs. \&11.

\section{CONCLUSION}

We have discussed the properties of long-lived resonances in hexagonally shaped dielectric microcavities. These microcavities play an important role for experiments on a class of microcrystal lasers [1, 2].

The numerical analysis revealed the following facts: (i) The resonance positions in the complex plane are approximated by a hyperbola. This fact is relevant for future experiments on microcrystal lasers because it implies that the laser threshold is lower for larger crystals. (ii) The near-field intensity pattern show a whispering-gallery-like structure. (iii) The emission is at corners in agreement with the experiments. (iv) The emission is directed. The high-intensity directions are not parallel to the facets. (v) The lifetimes and the emission directionality are sensitive to rounding of the corners.

The numerical analysis does not cover the full experimental regime. To overcome this limitation, we have introduced a semiclassical approximation which can be easily extended to any cavity of regular polygonal geometry. Our semiclassical ray model contains two new emission mechanisms: leakage due to boundary waves and due to the pseudointegrable ray dynamics. Explicit expressions for the resonance positions can be given even though the system is not integrable. The semiclassical approximation explains all numerical findings (i)-(v) in an intuitive way, except the emission directionality. To describe the directionality properly it is necessary to consider corner diffraction in the future.

Our results are not only relevant for microcrystal lasers but also for other kinds of polygonal-shaped microlasers. To name a few: hexagonal lasers with attached optical waveguides based on semiconductor heterostructures [30], hexagonally shaped solid polymer dye microcavities 31, equilateral-triangular laser cavities fabricated from semiconductor heterostructures [32], and square laser micropillar cavities based on dye-doped polymers [33].

\section{Acknowledgments}

I would like to thank S. W. Kim, T. Harayama, H. Schomerus, M. Hentschel, F. Laeri, and J. U. Nöckel for discussions. The work was supported by the Volkswagen foundation (project "Molekularsieblaser-Konglomerate im Infraroten").

\section{APPENDIX A: ANGULAR SHIFT}

In this section we discuss the semiclassical deviation of the angle of incidence from $60^{\circ}$. Let us first investigate -- and ++ -modes. Along the boundary, these modes are periodic with period $3 R$, i.e. $\psi(s+3 R)=\psi(s)$, where the arclength $s \in[0,6 R)$ parametrizes the boundary. Hence, the wave function along the boundary can be expanded as the following Fourier series

$$
\psi(s)=\sum_{M=-\infty}^{\infty} A_{M} \exp \left(i \frac{2 \pi}{3 R} M s\right) .
$$

From this we see that the conjugate momentum to $s$ or in other words the momentum component parallel to the boundary is "quantized" according to

$$
n \operatorname{Re}(k) \sin \theta=\frac{2 \pi}{3 R} M
$$

with integer $M$. Inserting the quantization condition (17) leads to

$$
\sin \theta=\sqrt{3} \frac{M}{m+\beta} .
$$

Linearising this equation around $\theta \approx \theta_{i}=60^{\circ}=\pi / 3$ yield in the semiclassical regime $m \gg \beta$

$$
\Delta \theta=2 \sqrt{3}\left(\frac{M}{m}-\frac{1}{2}\right)-2 \sqrt{3} \frac{M}{m} \frac{\beta}{m} .
$$

The smallest $\Delta \theta$ is realized for $M=m / 2(m$ is an even integer for -- and ++ -modes, see Tab. [1),

$$
\Delta \theta=-\sqrt{3} \frac{\beta}{m}=-\frac{2 \pi \beta}{3 n \operatorname{Re}(k R)} .
$$

Analogous arguments concerning -+ and +- -modes give the same result as in Eq. A5). 


\section{APPENDIX B: BOUNDARY-WAVE LEAKAGE}

In this section we compute the leakage due to boundary waves. To estimate the loss we consider the total internal reflection $\left(\theta_{i}>\theta_{c}\right)$ of a plane wave at an infinitely extended dielectric interface. This consideration is justified if $\operatorname{Re}(k R) \gg 1$ and $\theta_{i}$ not to close to the critical angle for total internal reflection $\theta_{c}$. Since $\theta_{e}$ is a complex number in Eq. (11), the wave in the lower-index region is evanescent, i.e. it decays exponentially with increasing distance from the boundary. Along the boundary the evanescent wave propagates with constant velocity. The corresponding total energy (or better intensity) flux at a given point at the boundary can be computed by means of

$$
\sigma=\int_{0}^{\infty} S_{x} d y
$$

where $S_{x}$ is the $x$-component of the Poynting vector 13

$$
\mathbf{S}=\frac{c}{8 \pi} \operatorname{Re}\left(\mathbf{E} \times \mathbf{H}^{*}\right)=-\frac{c}{8 \pi k} \operatorname{Re}\left(i \psi \nabla \psi^{*}\right) .
$$

Integration of Eq. (B1) using Eqs. (B2) and (11) yields

$$
\sigma=\frac{c}{4 \pi} \frac{n \sin \theta_{i}}{k \sqrt{n^{2} \sin ^{2} \theta_{i}-1}} \frac{1}{1+\alpha^{2}},
$$

with $\alpha$ from Eq. (15).

Now we relate the total flux to the intensity inside the resonator. The intensity of the wave function $\psi_{i}+\psi_{r}$ per area can be easily calculated to be $1 / 4 \pi$. Assuming that the boundary waves fully leave the cavity at corners, the outgoing relative intensity per unit time is given by

$$
\Delta I_{\mathrm{bw}}=\frac{c}{A} \frac{n \sin \theta_{i}}{k \sqrt{n^{2} \sin ^{2} \theta_{i}-1}} \frac{\chi}{1+\alpha^{2}},
$$

where $\chi$ is the number of corners (where the light is emitted), $A$ is the area of the resonator covered by the family of long-lived rays. Formula (B4) is valid for any regular polygon. For the hexagon, $\chi=6$ and $A$ is given by the area of the hexagon minus the region not accessible by the family of long-lived rays as depicted in Fig. 17. Elementary geometry yields

$$
A=\frac{3 \sqrt{3}}{2}\left(R^{2}-R_{\mathrm{c}}^{2}\right)=\sqrt{3} R^{2}
$$

The final result for the hexagon is then given in Eq. (21).

\section{APPENDIX C: PSEUDOINTEGRABLE LEAKAGE}

Here we compute the pseudointegrable leakage. We put the angular shift $\Delta \theta$ from Eq. (A5) into the initial conditions for the ray dynamics. Elementary geometry, see Fig. 12, shows that after one round trip, i.e. 6

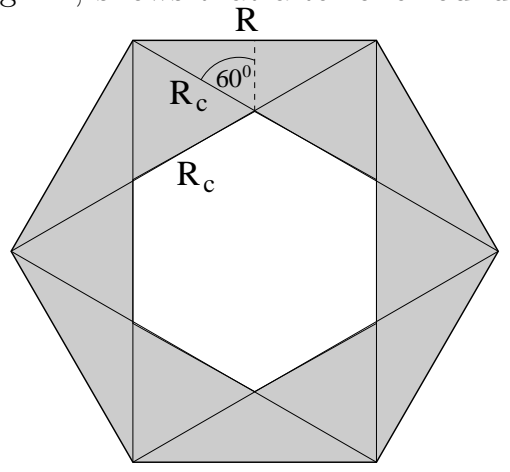

FIG. 17: Region (empty hexagon) not accessible by the family of long-lived rays. The region is bounded by a hexagonal "caustic" with side length $R_{\mathrm{c}}=R / \sqrt{3}$.

bounces, the angular deviation gives rise to the spatial deviation $\Delta s=|2 l \Delta \theta|$. The time for each round trip is $\Delta t=\ln / c$.

From Fig. 12 it is clear that points on the boundary within the distance $\Delta s$ from a corner leave the cavity after the next round trip. Hence, the relative outgoing intensity can be computed as

$$
\Delta I_{\mathrm{p}}=\frac{1}{R} \frac{\Delta s}{\Delta t}=\frac{4 \pi c}{3 \operatorname{Re}(k) R^{2}} \frac{\beta(n)}{n^{2}} .
$$

[9] J. Wiersig, Phys. Rev. E 64, 026212 (2001)

[1] U. Vietze et al., Phys. Rev. Lett. 81, 4628 (1998).

[2] I. Braun et al., Appl. Phys. B: Lasers Opt. 70, 335 (2000).

[3] J. Wiersig, e-print arXiv:physics/0206018 (2002).

[4] P. J. Richens and M. V. Berry, Physica D 2, 495 (1981).

[5] A. Hobson, J. Math. Phys. 16, 2210 (1975).

[6] A. Zemlyakov and A. Katok, Math. Notes 18, 760 (1975).

[7] R. Artuso, I. Guarneri, and L. Rebuzzini, Chaos 10, 189 (2000).

[8] J. Wiersig, Phys. Rev. E 62, R21 (2000).
[10] E. B. Bogomolny, U. Gerland, and C. Schmit, Phys. Rev. E 59, R1315 (1999).

[11] T. Harayama, P. Davis, and K. S. Ikeda, preprint 2002 (unpublished).

[12] T. Harayama, P. Davis, and K. S. Ikeda, Phys. Rev. Lett. 82, 3803 (1999).

[13] J. D. Jackson, Klassische Elektrodynamik (Walter de Gruyter, Berlin, New York, 1983). 
[14] R. H. Landau, Quantum Mechanics II, 2 ed. (John Wiley \& Sons, New York, 1996).

[15] L. M. Cureton and J. R. Kuttler, J. Sound Vib. 220, 83 (1999).

[16] J. U. Nöckel and A. D. Stone, in Optical Processes in Mircocavities, Vol. 3 of Advanced Series in applied Physics, edited by R. Chang and A. Campillo (World Scientific, Singapore, 1995).

[17] P. M. van den Berg and J. T. Fokkema, IEEE Trans. Antennas Propag. AP-27, 577 (1979).

[18] J. U. Nöckel, private communication (unpublished).

[19] M. Hentschel, H. Schomerus, and R. Schubert, e-print arXiv:physics/0208006 (2002).

[20] J. U. Nöckel and A. D. Stone, Nature 385, 45 (1997).

[21] E. Narimanov, G. Hackenbroich, P. Jacquod, and A. D. Stone, Phys. Rev. Lett. 83, 4991 (1999).

[22] A. K. Bhowmik, Appl. Opt. 39, 3071 (2000).

[23] M. C. Gutzwiller, Chaos in Classical and Quantum Mechanics, Vol. 1 of Interdisciplinary Applied Mathematics
(Springer, Berlin, 1990).

[24] E. Gutkin, J. Stat. Phys. 83, 7 (1996).

[25] H. E. Tureci, H. G. L. Schwefel, A. D. Stone, and E. E. Narimanov, e-print arXiv:physics/0207003 (2002).

[26] M. Born and E. Wolf, Principles of Optics (Pergamon Press, Oxford, New York, 1959).

[27] J. W. Ra, H. L. Bertoni, and L. B. Felsen, SIAM J. Appl. Math. 24, 396 (1973).

[28] N. H. Tran et al., Opt. Lett. 20, 1233 (1995).

[29] A. W. Poon, F. Courvoisier, and R. K. Chang, Opt. Lett. 26, 632 (2001).

[30] S. Ando, N. Kobayashi, and H. Ando, Jpn. J. Appl. Phys. 34, L4 (1995).

[31] M. Sasaki, Y. Li, T. Akatu, Y. Fujii, and K. Hane, Jpn. J. Appl. Phys. 39, 7145 (2000).

[32] H. C. Chang et al., Phys. Rev. A 62, 013816 (2000).

[33] G. D. Chern, A. W. Poon, and R. K. Chang, proceedings QELS (unpublished). 